Research Article

\title{
Ehresmann Semigroups from a Range Restriction Viewpoint
}

\author{
Wadii Hajji \\ Department of Mathematics and Natural Sciences, Prince Mohammad Bin Fahd University, Dhahran 34754, Saudi Arabia
}

Correspondence should be addressed to Wadii Hajji; whajj083@gmail.com

Received 28 June 2020; Accepted 21 April 2021; Published 11 May 2021

Academic Editor: Li Guo

Copyright (C 2021 Wadii Hajji. This is an open access article distributed under the Creative Commons Attribution License, which permits unrestricted use, distribution, and reproduction in any medium, provided the original work is properly cited.

The first theorem in this article provides the connection between Ehresmann semigroups and range prerestriction semigroups defined by the author. By this connection, we can redefine any Ehresmann semigroups by two unary operations and eight axioms. This connection leads us to a generalization of Ehresmann's theorem for a range prerestriction categories; as special cases, we obtain Ehresmann's theorems for range restriction categories and for inverse categories.

\section{Introduction}

This paper establishes the link between several classes of semigroups defined in semigroup theory and certain kinds of categories, namely, the category of partial maps and the category of partial relations. Our first theorem is the equivalence between Ehresmann semigroups defined by Lawson in [1] and range prerestriction semigroups defined by the author. A range prerestriction semigroup is defined to capture the notion of partiality on the domain and on the range of a partial relation on a set $(\operatorname{Rel}(X))$ which will denote the set of all relations between the set $(X)$. As a corollary of this theorem, we obtain the equivalence between range restriction semigroups and Ehresmann semigroups with an extra condition. Victoria Gould gave a more precise and general overview about these kind of semigroups $[2,3]$. The equivalence of inverse semigroups and inductive ordered groupoids was known to Ehresmann. In order to construct an inverse semigroup from an inductive ordered groupoid, we need to define the "pseudoproduct" of elements of an ordered groupoid. Ehresmann was interested in ordered categories not just ordered groupoids. Lawson in [1] attempts to answer the following question: what partial order does one need on a category in order to define an associative pseudoproduct? This generalization of Ehresmann theorem led us to our generalization at the categorical level. Therefore, we are going to add new theorems to the category of partial maps' range restriction category defined by Cockett and Guo [4] in 2004 and the category of partial relations range prerestriction category defined by the author.

Independently, in 1967, Sklar and Schweizer [5] found an axiomatic characterization of (the set of all partial functions on a set $(X)$. They found a class of semigroups with seven axioms, called function semigroups. We remark that there is a connection between range restriction semigroups and function semigroups. The range restriction categories with one object are exactly function semigroups; for the proof of this remark, see [6].

\section{Preliminaries}

We begin these preliminaries with an equivalent definition of a category where we omit the set of objects.

Definition 1. A category is a set $\mathscr{C}$, equipped with a partial multiplication satisfying the following axioms:

(i) $\left(C_{1}\right) x(y z)$ is defined if and only if $(x y) z$ is defined in this case and they are equal

(ii) $\left(C_{2}\right) x y$ and $y z$ are defined and then $(x y) z$ is defined

(iii) $\left(C_{3}\right)$ For each $x \in \mathscr{C}$, there exists an identity $e$ and an identity $f$ such that $x e=x$ and $f x=x$

One can prove that the identity $e$ (resp. $f$ ) such that $x e=$ $x$ (resp. $f x=x$ ) is unique and call the domain of $x$, denoted by $d(x)$ (resp. range or codomain of $x$, denoted by $r(x)$ ). 
Definition 2. A category $\mathscr{C}$ is ordered if it satisfies the following axioms:

$$
\begin{aligned}
& \left(O C_{1}\right)(\mathscr{C}, \leqslant) \text { is a poset } \\
& \left(O C_{2}\right) x \leqslant y \text { implies } r(x) \leqslant r(y) \text { and } d(x) \leqslant d(y) \\
& \left(O C_{3}\right) \text { If } x^{\prime} \leqslant x \text { and } y^{\prime} \leqslant y \text { and both } x^{\prime} y^{\prime} \text { and } x y \text { exist, } \\
& \text { then } x^{\prime} y^{\prime} \leqslant x y \\
& \left(O C_{4}\right) \text { If } r(x)=r(y) \text { and } d(x)=d(y) \text { and } x \leqslant y \text {, then } \\
& x=y \text { (that is, } \leqslant \text { is trivial on hom-sets) }
\end{aligned}
$$

We can view an ordered category as an internal category in poset (the category which has posets for objects and ordered-preserving maps as arrows) with additional condition (see Proposition 4.3.3 in [6]).

Definition 3. Let $\mathscr{C}$ be an ordered category and $\mathscr{C}_{0}$ the set of identities:

(1) $\mathscr{C}$ has restrictions if every $x \in \mathscr{C}$ and $e \leqslant d(x)$, where $e \in \mathscr{C}_{0}$, then there exists a unique element called the restriction of $x$ to $e$, which will be denoted by $(x \mid e)$, such that $(x \mid e) \leqslant x$ and $d(x \mid e)=e$

(2) $\mathscr{C}$ has corestrictions if every $x \in \mathscr{C}$ and $e \leqslant r(x)$, where $e \in \mathscr{C}_{0}$, then there exists a unique element called the corestriction of $x$ to $e$, which will be denoted by $(e \mid x)$, such that $(e \mid x) \leqslant x$ and $r(e \mid x)=e$

To a category theorist, a restriction condition in an ordered category is equivalent to saying that the map d: $\mathscr{C} \longrightarrow \mathscr{C}_{0}$ is a discrete fibration (we consider $\mathscr{C}$ as a poset category) [6].

\section{Semigroups}

In this section, we give the definition and properties of each of the classes of semigroups that we shall consider, namely, Ehresmann semigroups and range prerestriction semigroups and the link between them in Theorem 2. As a corollary, we prove that the range restriction semigroups are precisely Ehresmann semigroups with an additional condition.

3.1. Ehresmann Semigroups. This section follows Lawson [1] and Gomes and Gould [7]. Let $S$ be a semigroup and let $U \subseteq E(S)$ be a fixed, nonempty subset of the idempotents of $S$. Before starting our definitions, we define two binary relations:

$$
\begin{aligned}
& (x, y) \in \tilde{\mathscr{L}}, \quad \text { if for all } e \in U, \text { we have } x e=x \Longleftrightarrow y e=y, \\
& (x, y) \in \widetilde{\mathscr{R}}, \quad \text { if for all } e \in U, \text { we have } e x=x \Longleftrightarrow e y=y .
\end{aligned}
$$

Remark 1 . The binary relations $\widetilde{\mathscr{L}}$ and $\widetilde{\mathscr{R}}$ are equivalence relations on $S$.

Notation 1. Let $x$ be an element in $S$. We denote the classes $\widetilde{\mathscr{L}}-$ and $\widetilde{\mathscr{R}}$-classes containing the element $x$ by $\widetilde{L_{x}}$ and $\widetilde{R_{x}}$, respectively.
Definition 4. Let $S$ be a semigroup. An equivalence relation $\rho$ on $S$ is a right (resp. left) congruence if and only if every $(a, b) \in \rho$ and $c \in S$ imply $(a c, b c) \in \rho(\operatorname{resp} .(c a, c b) \in \rho)$.

Definition 5. Let $S$ be a semigroup. Then, $(S, U)$ is an Ehresmann semigroup if $U$ is a commutative subsemigroup of $S$ and if each $\widetilde{\mathscr{R}}$-class and each $\widetilde{\mathscr{L}}$-class contains at least one element of $U$. If $\widetilde{\mathscr{L}}$ is a right congruence and $\widetilde{\mathscr{R}}$ is a left congruence, in this case, we say that $S$ has the congruence condition.

Now, we provide a useful proposition to establish the equivalence between Ehresmann semigroups and range prerestriction semigroups.

Proposition 1. Let $(S, U)$ be an Ehresmann semigroup. Then,

(1) Each $\widetilde{\mathscr{L}}$ and $\widetilde{\mathscr{R}}$ class contains a unique element in $U$. Let $x$ be in $S$. We denote by $\bar{x}$ (resp. $\widehat{x}$ ) the unique element of $U \cap \widetilde{L_{x}}\left(\operatorname{resp} . U \cap \widetilde{R_{x}}\right)$.

(2) For each $e \in U$, both $\bar{e}=e$ and $\widehat{e}=e$.

(3) The congruence condition in $S$ is equivalent to these two axioms:

$$
\begin{aligned}
& \overline{x y}=\overline{\bar{x} y}, \\
& \widehat{x y}=\widehat{x \hat{y}} .
\end{aligned}
$$

Proof

(1) We will prove the statement for $\widetilde{\mathscr{L}}$. The proof for $\widetilde{\mathscr{R}}$ is similar. Let $e, f \in U$ such that $e \tilde{\mathscr{L}} f$. Then, $e f=e$ and $f e=f$. As $U$ is a commutative sub-semigroup, $e=e f=f e=f$.

(2) Too trivial because each class contains one idempotent element in $U$.

(3) Suppose that $S$ satisfies the congruence condition. Let $x$ be in $S$; then, $x \widetilde{\mathscr{L}} \bar{x}$. Then, for any $y \in S$, we have $x y \tilde{\mathscr{L}} \bar{x} y$; therefore, $\overline{x y}=\overline{\bar{x} y}$.

Now, suppose that $\overline{x y}=\overline{\bar{x} y}$ holds for all $x, y \in S$. Suppose that $x \widetilde{\mathscr{L}} y$, and let $z$ be in $S$. Then, $\bar{x}=\bar{y}$ so $\bar{x} z=\bar{y} z$; hence, $\overline{x z}=\overline{\bar{x} z}=\overline{\bar{y} z}=\overline{y z}$. Therefore, $x z \tilde{\mathscr{L}} y z$. The second axiom is dual.

3.2. Range Prerestriction Semigroups. Manes, in [8], introduced guarded semigroups as a restriction category with one object and without necessarily an identity. Restriction categories were defined by Cockett and Lack in [9] to capture the notion of partiality on the domain of partial functions. Following similar ideas, we define range restriction semigroup as a range restriction category (defined by Cockett and Guo [4] to capture the notion of partiality not only on the domain but also on the range) with one object and without necessarily the identity.

In 2005, Manes and Cockett defined prerestriction categories to capture the notion of partiality on the domain 
of partial relations in the category of sets and partial relations. Following similar ideas to Manes and Cockett, we now define range prerestriction semigroups, which have the almost the same axioms as range restriction semigroups, except for one condition $R 4$, which we weaken.

Recall from [4] that a range restriction semigroup is a semigroup with two unary operations and which satisfy the following axioms:
(i) $(R 1) x \bar{x}=x$
(ii) $(R 2) \overline{x y}=\overline{y x}$
(iii) $(R 3) \overline{x \bar{y}}=\overline{x y}$
(iv) $(R 4) \bar{x} y=y \overline{x y}$
(v) $(R R 1) \overline{\hat{x}}=\hat{x}$
(vi) (RR2) $\hat{x} x=x$
(vii) $(R R 3) \widehat{\bar{y} x}=\bar{y} \hat{x}$
(viii) (RR4) $\widehat{y \widehat{x}}=\widehat{y x}$

\section{Example 1}

(i) Let $X$ be a set and $P f n(X)$ the semigroup of all partial functions on $X$. It is a range restriction semigroup. Indeed, for any element $f$ in $P f n(X) f: X \longrightarrow X$, the restriction structure is given by $\bar{f}=1_{\operatorname{dom}(f)}$ and the range structure $\widehat{f}=1_{\operatorname{ran}(f)}$.

(ii) Any monoid with identity $e$ has trivial restriction structure and trivial range structure given by

$$
\bar{f}=\widehat{f}=e .
$$

However, the problem is that $R 4$ is not valid in $\operatorname{Rel}(X)$. So, instead of $R 4$, we introduce $p R 4 \overline{x y}=\overline{\bar{x} y}$. This axiom is true in $\operatorname{Rel}(X)$.

Now, we can define officially a range prerestriction semigroup as follows.

Definition 6. A semigroup $S$ is a range prerestriction semigroup if and only if there are two unary operations and satisfying the following axioms:
(i) $(R 1) x \bar{x}=x$
(ii) $(R 2) \overline{x y}=\overline{y x}$
(iii) $(R 3) \overline{x \bar{y}}=\overline{x y}$
(iv) $(p R 4) \overline{x y}=\overline{\bar{x} y}$
(v) $(R R 1) \overline{\hat{x}}=\widehat{x}$
(vi) (RR2) $\widehat{x} x=x$
(vii) $(R R 3) \widehat{\bar{y} x}=\bar{y} \hat{x}$
(viii) $(R R 4) \widehat{y \hat{x}}=\widehat{y x}$

Proposition 2. A range restriction semigroup is a range prerestriction semigroup.
Proof. $\overline{\bar{x} y}=\overline{y \overline{x y}}=\bar{y} \overline{x y}=\overline{x y} \bar{y}=\overline{x y \bar{y}}=\overline{x y}$ using R4, R2, $R 3$, and $R 1$.

From this proposition, we can deduce that all the proprieties for range prerestriction semigroups remain true for range restriction semigroups. Some basic properties of range prerestriction semigroups are recorded in the following proposition.

Proposition 3. Let $S$ be a range prerestriction semigroup. Then, for every element $x$ and $y$ in $S$, we have the following:

(1) $\bar{x}$ is an idempotent element

(2) $\bar{x} \overline{y x}=\overline{y x}$

(3) $\overline{\bar{x}}=\bar{x}$

(4) $\overline{\overline{y x}}=\overline{y x}$

(5) $x \bar{y}=x \Leftrightarrow \bar{x}=\overline{x y}$

(6) $\hat{x} \hat{y}=\hat{y} \hat{x}$

(7) $\hat{x} \bar{y}=\bar{y} \hat{x}$

(8) $\widehat{x} y=\widehat{x} \widehat{y}$

(9) $\hat{x}$ is an idempotent element

(10) $\widehat{\hat{x}}=\widehat{x}$

(11) $\widehat{\bar{x}}=\bar{x}$

(12) $\widehat{x y} \widehat{x}=\widehat{x y}$

(13) $\widehat{\widehat{x} \hat{y}}=\widehat{x} \widehat{y}$

(14) $\hat{x} y=y \Leftrightarrow \hat{y}=\hat{x} \hat{y}$

Proof

(1) By $R 3$ and $R 1$, we have $\overline{x x}=\overline{x \bar{x}}=\bar{x}$.

(2) By $R 2, \quad R 3$, and $R 1$, we have $\bar{x} \overline{y x}=\overline{y x} \bar{x}=\overline{y x \bar{x}}=\overline{y x}$.

(3) By (1), R3, R2, $\quad R$ and $R 1$, we have $\overline{\bar{x}}=\overline{\overline{x x}}=\overline{\bar{x} x}=\overline{x \bar{x}}=\bar{x}$.

(4) By $R 3, \overline{\bar{y}} \bar{x}=\overline{\bar{y}} \bar{x}=\bar{y} \bar{x}$.

(5) Suppose that $x \bar{y}=x$. By R3, we get $\bar{x}=\overline{x \bar{y}}=\overline{x y}$. Suppose that $\bar{x}=\overline{x y}$. And, by $R 1$, we have $x \bar{x}=x$. Therefore, $x \overline{x y}=x$; thus, $x \bar{y}=x$.

(6) By $R R 1$ and $R 2, \hat{x} \hat{y}=\overline{\hat{x}} \hat{y}=\overline{\hat{y}} \widehat{x}=\hat{y} \hat{x}$.

(7) By $R R 1$ and $R 2, \hat{x} \bar{y}=\overline{\hat{x}} \bar{y}=\bar{y} \bar{x}=\bar{y} \hat{x}$.

(8) By $R R 1$ and $R R 3, \widehat{\widehat{x} y}=\widehat{\widehat{x} y}=\overline{\hat{x}} \hat{y}=\widehat{x} \hat{y}$.

(9) By $R R 1$ and (1), $\hat{x} \widehat{x}=\overline{\hat{x}} \hat{x}=\overline{\hat{x}}=\hat{x}$.

(10) By $R R 2, R R 4$, and (9), $\widehat{\hat{x}}=\widehat{\widehat{x} \hat{x}}=\widehat{\widehat{x} x}=\widehat{x}$.

(11) From $R R 2$, we have $\hat{x} x=x$ for each $x \in S$. In particular, we get $\widehat{\bar{x} x}=\bar{x}$. Now, we can use (7) $\bar{x} \hat{x}=\widehat{\bar{x}} \bar{x}=\bar{x}$. Thus, using $R R 3, \widehat{\overline{x x}}=\overline{x \hat{x}}=\bar{x}$. An application of (1) gives $\widehat{\bar{x}}=\bar{x}$.

(12) By (6), (8), and $R R 2, \widehat{x y} \hat{x}=\widehat{x} \widehat{x y}=\widehat{\widehat{x} x y}=\widehat{x y}$.

(13) By RR1, RR2, and (10), $\widehat{\widehat{x} \hat{y}}=\widehat{\widehat{\hat{x}} \hat{y}}=\overline{\hat{x}} \widehat{\hat{y}}=\widehat{x} \hat{y}$.

(14) Suppose that $\hat{x} y=y$. By (8), we get $\widehat{y}=\widehat{\widehat{x} y}=\hat{x} \hat{y}$. Suppose now that $\hat{y}=\hat{x} \hat{y}$. By $R R 2$, we have $\hat{y} y=y$. Therefore, $\hat{x} \hat{y} y=y$; thus, $\hat{x} y=y$. 
We shall use the properties established in Proposition 3 without further comment.

3.3. Ehresmann Semigroups Are Equivalent to Range Prerestriction Semigroups. Let $S$ be a range prerestriction semigroup. To distinguish between idempotent elements and the elements of the form $\bar{x}$ and $\hat{x}$, where $x \in S$, we will introduce the following definition.

Definition 7. Let $S$ be range prerestriction (range restriction) semigroup. An element $s$ in $S$ is a restriction idempotent if there is an element $x$ in $S$ such that $s=\bar{x}$.

Theorem 1. In a given range prerestriction (range restriction) semigroup $S$, the following statements are equivalent:

(1) $s$ is a restriction idempotent

(2) $s=\widehat{s}$

(3) There is an element $x$ in $S$ such that $s=\widehat{x}$

(4) $s=\bar{s}$

Proof. If $s$ is a restriction idempotent, then $s=\bar{x}$ for some $x$ in $S$. Hence,

$$
\widehat{s}=\widehat{\bar{x}}=\bar{x}=s .
$$

Thus, (1) implies (2). The remaining implications are trivial.

An idempotent and commutative semigroup is called a semilattice.

Lemma 1. In range prerestriction (range restriction) semigroups, the set of all restriction idempotent forms a semilattice.

Proof. The proof of this theorem is trivial because, for every two restriction idempotent $s$ and $t$ in $S$, we have that $s=\bar{s}$ and $t=\bar{t}$, and these two elements commute using $R 2$. The fact that they form a semigroup is again obvious using $R 3$.

Theorem 2. Let $S$ be a semigroup. Then, $S$ is an Ehremann semigroup if and only if $S$ is a range prerestriction semigroup.

Proof. Let $S$ be a range prerestriction semigroup and let $U$ be the set of all restriction idempotents. By Lemma $1, U$ is a commutative subsemigroup of $S$. Each $\widetilde{\mathscr{L}}$-class contains at least one element; indeed, $(x, \bar{x}) \in \widetilde{\mathscr{L}}$ because, for all $e \in U$, we have $x e=x \Longleftrightarrow \bar{x} e=\bar{x}$. Dually, we can prove $(x, \widehat{x}) \in \widetilde{\mathscr{R}}$ too, i.e., we can prove that, for all $e \in U$, we have $e x=x \Longleftrightarrow e \hat{x}=\hat{x}$. By Proposition 1 (3), we conclude that any range prerestriction semigroup is Ehresmann semigroup by taking the set $U$ to be all the restriction idempotents.

Conversely, suppose now that $(S, U)$ is an Ehresmann semigroup. Therefore, for every element $x$ in $S$, there exist unique elements (using Proposition 1) $\bar{x}$ and $\hat{x}$ in $U$ such that $x \bar{x}=x$ and $\hat{x} x=x$; thus, $R 1$ and $R R 2$ are verified. Now, from Proposition 1 (2), for every element $e$ in $U, \bar{e}=e$ and $\widehat{e}=e$. In particular, when $e=\widehat{x}$, we get $\overline{\hat{x}}=\widehat{x}$; thus, $R R 1$ is satisfied. From Proposition 1 (3), S satisfies the congruence condition, so $\overline{x y}=\overline{\bar{x} y}$ and $\widehat{x y}=\widehat{x \hat{y}}$. Therefore, $p R 4$ and $R R 4$ hold.

Now, for all elements $x$ and $y$ in $S, \bar{x}$ and $\bar{y}$ are in $U$, so by definition, they commute; thus, $R 2$ is verified.

To prove $R 3$, we have to use a particular case of $p R 4$ $\overline{x y}=\overline{\bar{x} y}$, namely, $\overline{x \bar{y}}=\overline{\overline{x y}}$. Then, $\overline{\overline{x y}}=\overline{x y}$ because $\overline{x y}$ belongs to $U$ ( $U$ is a semigroup). So, by Proposition 1 (2), for every element $e$ in $U, \bar{e}=e$ thus $R 3$. It remains to show $R R 3$ $\widehat{\bar{y} x}=\bar{y} \hat{x}$; for that, we are going to use $R R 4$. By $R R 4$, we have $\widehat{y} x=\widehat{y \hat{x}}$ which is equal to $\bar{y} \widehat{x}$ ( $U$ is a semigroup, and by Proposition 1 (2), for every element $e$ in $U, \widehat{e}=e$ ).

Now, we have obtained this connection between Ehresmann semigroups and range prerestriction semigroups, and we can redefine Ehresmann semigroups by eight axioms. In particular, Ehresmann semigroups form a variety of semigroups with two unary operations, so free Ehresmann semigroups exist.

We can easily pass to the categorical level and generalize several theorems. In this paper, we will give the generalizations for the following theorem:

Theorem 3. (see Theorem 4.24 in [1]). The category of Ehresmann semigroups and admissible homomorphisms in the sense of Lawson [1] is isomorphic to the category of Ehresmann categories and strongly ordered functors.

Remark 2. From the fact that Ehresmann semigroups are range prerestriction semigroups, we can easily check the following result: admissible homomorphisms are equivalent to semigroups' homomorphisms preserving the two unary operations and?

Definition 8. We define an Ehresmann category to be a category $\mathscr{C}$ equipped with two relations $\leqslant_{l}$ and $\leqslant_{r}$, satisfying the following axioms:

$\left(E_{1}\right)\left(\mathscr{C}, \leqslant_{r}\right)$ is an ordered category with corestrictions $\left(E_{2}\right)\left(\mathscr{C}, \leqslant_{l}\right)$ is an ordered category with restrictions

$\left(E_{3}\right)$ If $e, f \in \mathscr{C}_{0}$, then $e \leqslant_{r} f \Longleftrightarrow e \leqslant_{l} f$, and put $\leqslant=\leqslant_{r}=$ $\leqslant l$ on $\mathscr{C}_{0}$

$\left(E_{4}\right)\left(\mathscr{C}_{0}, \leqslant\right)$ is a meet semilattice

$\left(E_{5}\right) \leqslant_{r} \circ \leqslant_{l}=\leqslant_{l} \circ \leqslant_{r}$, andwe define the relation $\leqslant_{e}$ equal to $\leqslant_{r} \circ \leqslant_{l}$

$\left(E_{6}\right)$ If $f \leqslant_{r} y$ and $f \in \mathscr{C}_{0}$, then $(f \mid d(f) \wedge f) \leqslant_{r}$ $(y \mid d(y) \wedge f)$

$\left(E_{7}\right)$ If $f \leqslant_{l} y$ and $f \in \mathscr{C}_{0}$, then $(r(f) \wedge f \mid f) \leqslant_{l}$ $(r(y) \wedge f \mid y)$

As mentioned above, a range restriction semigroup is exactly an Ehresmann semigroup (range prerestriction semigroup) with an extra condition which is $R_{4}$. Therefore, in the next section, we will give Ehresmann's theorem for range restriction semigroups using this later fact.

3.4. Range Restriction Semigroups. We will state, as a corollary of Theorem 3, the isomorphism between range 
restriction semigroups and $R_{4}$-Ehresmann categories which are Ehresmann categories with a condition, which we will define later.

Now, we can define three different orders on any range prerestriction (range restriction) semigroup following Lawson (Proposition 3.13 in [1]):

(i) $x \leqslant_{e} y \Longleftrightarrow$, and there exist $e, f \in U$ such that $x=e y f$

(ii) $x \leqslant_{r} y \Longleftrightarrow$, and there exists $e \in U$ such that $x=e y$

(iii) $x \leqslant_{l} y \Longleftrightarrow$, and there exists $f \in U$ such that $x=y f$

Let $S$ be an Ehresmann (range Prerestriction) semigroup. Then, $R 4$ (which is equivalent to Lawson's $I C_{r}$ condition [1]) holds if and only if $\leqslant_{r} \subseteq \leqslant_{l}$. Indeed, let $x \leqslant_{r} y$. Then, there exists $e \in U$ such that $x=e y$. By applying the $R_{4}$ condition, we get that $x=e y=y \overline{e y}$; thus, $x \leqslant_{l} y$. The converse is clear; see also Lemma 3.15 in [1]. Therefore, we have to add this condition to our definition of Ehresmann category to get an $R_{4}$-Ehresmann category $\mathscr{C}$. And, we have more than that, and the relation $\leqslant_{l}$ is compatible with the multiplication, i.e., for every elements $x, y, u$, and $v$ in $\mathscr{C}$ such that $x \leqslant_{l} y$ and $u \leqslant_{l} v$; then, $x u \leqslant_{l} y v$. We will prove this last statement using two lemmas. First, let us define a $R_{4}$-Ehresmann category.

Definition 9. An $R_{4}$-Ehresmann category is an Ehresmann category with $R_{4} E$ :

$$
\leqslant_{l}=\leqslant_{r} \circ \leqslant_{l}=\leqslant_{l} \circ \leqslant_{r}
$$

To deduce Corollary 1, which is another version of Ehresmann's theorem, but now, for an range restriction semigroups, we need the following lemmas. The first lemma is proved just to prove the second lemma.

Lemma 2 (see Lemma 4.4 in [1]). Let $\mathscr{C}$ be an Ehresmann category:

(1) If $e \leqslant d(x)$ and $f \leqslant e$, then $(x \mid f) \leqslant \leqslant_{l}(x \mid e) \leqslant l$

(2) If $e \leqslant r(x)$ and $f \leqslant e$, then $(f \mid x) \leqslant{ }_{r}(e \mid x) \leqslant{ }_{r} x$

For any $R_{4}$-Ehresmann category $\mathscr{C}$, we can define a pseudoproduct as follows. Let $x, y \in \mathscr{C}$ :

$$
x \otimes y=(x \mid e)(e \mid y), \quad \text { where } e=d(x) \wedge r(y) .
$$

The following lemma is similar to what is proved in Proposition 5.2 in [1], which states that, from a de Barros category, we can construct a de Barros semigroup.

Lemma 3. Let $\mathscr{C}$ be an $R_{4}$-Ehresmann category. Let $x, y$, and $u$ be elements in $\mathscr{C}$ :

(1) If $x \leqslant_{l} y$, then $x \otimes u \leqslant_{l} y \otimes u$

(2) If $x \leqslant \leqslant_{l} y$, then $u \otimes x \leqslant l u \otimes y$

That is, the relation $\leqslant_{l}$ is compatible with the multiplication.

Proof. Let us prove (1). Similarly, we can prove (2). First, we will prove (1) only for an element $e$ in $\mathscr{C}_{0}$, i.e., if $x \leqslant l y$, then $x \otimes e \leqslant l y \otimes e$, for some $e \in \mathscr{C}_{0}$. Since $x \leqslant l$, then $d(x) \leqslant d(y)$; thus, $d(x) \wedge e \leqslant d(y) \wedge e$ from the lemma above $(y \mid d(x) \wedge e) \leqslant_{l}(y \mid d(y) \wedge e)$. Now, it is clear that $x \otimes e \leqslant_{l} y \otimes e$. Let $u$ be any element in $\mathscr{C}$. Therefore,

$$
\begin{aligned}
& x \otimes u=(x \mid e)(e \mid u), \quad \text { where } e=d(x) \wedge r(u), \\
& y \otimes u=(y \mid f)(f \mid u), \quad \text { where } f=d(y) \wedge r(u) .
\end{aligned}
$$

From $x \leqslant_{l} y$, we obtain $x \otimes r(u) \leqslant_{l} y \otimes r(u)$ which is just $(x \mid e) \leqslant l(y \mid f)$. From $x \leqslant l$, we have $d(x) \leqslant d(y)$ so $e \leqslant f \leqslant r(u)$. However, then by the previous lemma, we obtain $(e \mid u) \leqslant_{r}(f \mid u)$. However, $\leqslant_{r} \subseteq \leqslant_{l}$ so that $(e \mid u) \leqslant_{l}(f \mid u)$. From the fact that $d(x \mid e)=r(e \mid u)=e$ and $d(y \mid f)=r(f \mid u)=f$, we may conclude that

$$
(x \mid e)(e \mid u) \leqslant_{l}(y \mid f)(f \mid u)
$$

which is just $x \otimes u \leqslant y \otimes u$.

Corollary 1. The category of range restriction semigroups and their homomorphisms is isomorphic to the category of $R_{4}$-Ehresmann categories and strongly ordered functors.

\section{Range Prerestriction Categories}

We will give in this section some helpful definitions and propositions that will lead us to prove our main theorem in this paper (generalization of Ehresmann's Theorem).

We start this section by giving a categorical generalization of range prerestriction semigroups.

Definition 10. Let $\mathscr{C}$ be a small category. A range Prerestriction structure on a category $\mathscr{C}$ is an assignment to each arrow $x: d(x) \longrightarrow r(x)$, two arrows $\bar{x}: d(x) \longrightarrow d(x)$ and $\hat{x}: r(x) \longrightarrow r(x)$, such that the following eight conditions are satisfied:

$$
\begin{aligned}
& (R 1) x \bar{x}=x \text { for each map } x \\
& (R 2) \overline{x y}=\overline{y x} \text { with } d(x)=d(y) \\
& (R 3) \overline{y \bar{x}}=\overline{y x} \text { with } r(x)=r(y) \\
& (p R 4) \overline{y x}=\overline{\bar{y} x} \text { with } r(x)=d(y) \\
& (R R 1) \overline{\hat{x}}=\widehat{x} \text { for each map } x \\
& (R R 2) \hat{x} x=x \text { for each map } x \\
& (R R 3) \widehat{\bar{y} x}=\bar{y} \hat{x} \text { with } r(x)=d(y) \\
& (R R 4) \widehat{y \hat{x}}=\widehat{y x} \text { with } r(x)=d(y)
\end{aligned}
$$

If a category $\mathscr{C}$ has a range prerestriction structure, we call it a range prerestriction category.

If $\mathscr{C}$ satisfies the conditions $R 1, R 2, R 3$, and $p R 4$, we call this category a prerestriction category, see [10]. This notion is due to Manes and Cockett. As we mentioned in the preliminaries, we will omit the set of objects in any category, and this gives us an equivalent definition which is easy to work with. The properties below come from Proposition 1 for range prerestriction semigroups, which are still true for range prerestriction categories. We are not going to prove these properties because we can apply the same arguments as before. 
Proposition 4. Let $\mathscr{C}$ be a range prerestriction category and $x, y$ arrows in $\mathscr{C}$. We have the following:

(1) $\bar{x}$ is idempotent

(2) $\bar{x} \overline{y x}=\overline{y x}$ if $r(x)=d(y)$

(3) $\overline{\bar{x}}=\bar{x}$

(4) $\overline{\overline{y x}}=\overline{y x}$ if $d(x)=d(y)$

(5) $x \bar{y}=x \Leftrightarrow \bar{x}=\overline{x y}$ if $d(x)=d(y)$

(6) $\hat{x} \hat{y}=\hat{y} \hat{x}$ if $r(x)=r(y)$

(7) $\hat{x} \bar{y}=\bar{y} \hat{x}$ if $r(x)=d(y)$

(8) $\widehat{\widehat{x} y}=\widehat{x} \hat{y}$ if $r(x)=r(y)$

(9) $\hat{x} \hat{x}=\hat{x}$ for each map $x$

(10) $\widehat{\hat{x}}=\hat{x}$ for each map $x$

(11) $\widehat{\bar{x}}=\bar{x}$ for each map $x$

(12) $\widehat{y x} \hat{y}=\widehat{y x}$ if $r(x)=d(y)$

(13) $\widehat{\hat{y} \widehat{x}}=\widehat{y} \hat{x}$ if $r(x)=r(y)$

(14) $\hat{x} y=y \Leftrightarrow \hat{y}=\hat{x} \hat{y}$ if $r(x)=r(y)$

Definition 11. Let $\mathscr{C}$ be a range prerestriction (or restriction) category. An arrow $x$ in $\mathscr{C}$ is called a restriction idempotent if there is an arrow $y$ in $\mathscr{C}$ such that $x=\bar{y}$.

The following theorem, inspired from the work on function systems in [5], describes various equivalent versions of this definition.

Theorem 4. In a given range prerestriction category $\mathscr{C}$, the following statements are equivalent:

(1) $x$ is a restriction idempotent

(2) $x=\widehat{x}$

(3) There is an element $y$ in $\mathscr{C}$ such that $x=\hat{y}$

(4) $x=\bar{x}$

Proof. If $x$ is a restriction idempotent, then $x=\bar{y}$ for some arrow $y$ in $\mathscr{C}$. Hence,

$$
\widehat{x}=\widehat{\bar{y}}=\bar{y}=x \text {. }
$$

Thus, (1) implies (2). The remaining implications are trivial.

Notation 2. Let $\mathscr{C}$ be a range prerestriction (or range restriction) category, and $\alpha$ and $\beta$ two identities. We denote

(1) $\operatorname{Hom}(\alpha, \beta)=\{x \in \mathscr{C} \mid d(x)=\alpha$ and $r(x)=\beta\}$

(2) $\operatorname{RIHom}(\alpha, \alpha)=$

$\{x \in \operatorname{Hom}(\alpha, \alpha) \mid$ such that $x$ is a restriction idempotent $\}$

(3) $R I \mathscr{C}=\left\{\operatorname{RIHom}(\alpha, \alpha) \mid \alpha \in \mathscr{C}_{0}\right\}$, where $\mathscr{C}_{0}$ is the set of all identities in $\mathscr{C}$

Theorem 5. In a range prerestriction (or range restriction) category, the set RIHom $(\alpha, \alpha)$ is a idempotent commutative semigroup.
Proof. The proof of this theorem is trivial because for every two restriction idempotents $x$ and $y$ in $\mathscr{C}$, we have that $x=\bar{x}$ and $y=\bar{y}$ and these two elements commute using $R 2$. The fact that it forms a semigroup is again obvious using Proposition 4 (4).

Now, we introduce three relations which will help us to investigate the structure of range prerestriction and restriction categories. We remark that all the definitions and propositions for range prerestriction categories are still true for range restriction categories, but there are some properties which are true for range restriction but not for range prerestriction categories.

The following definition comes from Lawson [1], but is now formulated for range prerestriction categories.

Definition 12. Let $\mathscr{C}$ be range prerestriction (or range restriction) category. We can define three relations on any $\operatorname{Hom}(\alpha, \beta)$, where $\alpha$ and $\beta$ are identities as follows:

$$
\begin{aligned}
& \text { (i) } x \leqslant_{e} y \Leftrightarrow x=\hat{x} y \bar{x} \\
& \text { (ii) } x \leqslant_{r} y \Leftrightarrow x=\hat{x} y \\
& \text { (iii) } x \leqslant_{l} y \Leftrightarrow x=y \bar{x}
\end{aligned}
$$

Proposition 5. On a range prerestriction category $\mathscr{C}$, the relations $\leqslant_{e}, \leqslant_{r}$ and $\leqslant_{l}$ are partial orders on $\operatorname{Hom}(\alpha, \beta)$, where $\alpha$ and $\beta$ are identities. Furthermore, the three orders are the same on $\operatorname{RIHom}(\alpha, \alpha)$, which will be denoted $\leqslant$. Moreover, $\operatorname{RIHom}(\alpha, \alpha)$ with this order forms a meet semilattice with the top element $\alpha$. And, if we have

(i) $x \leqslant_{r} y$ and $\hat{x}=\hat{y}$, then $x=y$

(ii) $x \leqslant_{l} y$ and $\bar{x}=\bar{y}$, then $x=y$

Proof. We will prove that $\leqslant_{e}$ is a partial order on $\operatorname{Hom}(\alpha, \beta)$. The proof for the other relations is similar.

Reflexivity: clearly $x=\widehat{x} x \bar{x}$ so that $x \leqslant_{e} x$, where $x$ is an arrow in $\mathscr{C}$.

Antisymmetry: let $x \leqslant_{e} y$ and $y \leqslant_{e} x$. Then, $x=\hat{x} y \bar{x}$ and $y=\widehat{y} x \bar{y}$; thus,

$$
\begin{aligned}
\bar{x} & =\overline{\hat{x} y \bar{x}}=\overline{(\hat{x} y)} \bar{x}=\overline{(\widehat{x} \hat{y} x \bar{y}) \bar{x}}=\overline{(\widehat{x} \hat{y} x)} \overline{y x} \\
& =\overline{(\widehat{x} \hat{y} x)} \overline{x y}=\overline{(\hat{x} \hat{y} x \bar{x}) \bar{y}}=\overline{(\widehat{y} \hat{x} x)} \bar{y}=\overline{(\widehat{y} x)} \bar{y}=\overline{\hat{y} x \bar{y}}=\bar{y} .
\end{aligned}
$$

Similarly, we can prove $\hat{x}=\hat{y}$. Therefore, $x=\widehat{x} y \bar{x}=\widehat{y} y \bar{y}=y$.

Transitivity: if $x \leqslant_{e} y$ and $y \leqslant_{e} h$, then $x=\hat{x} y \bar{x}$ and $y=\hat{y} h \bar{y}$; furthermore, $x=\hat{x} y \bar{x}$ implies $\bar{x}=\overline{\hat{x}} y \bar{x}=\overline{(\hat{x} y)} \bar{x}=$ $\overline{(\hat{x} y \bar{y})} \bar{x}=\overline{(\hat{x} y)} \overline{y x}=\bar{y} \overline{(\widehat{x} y)} \bar{x}=\bar{y} \bar{x} y \bar{x}=\overline{y x}$. Similar to this proof, we can show $\hat{x}=\hat{x} \hat{y}$. Thus, $x=\hat{x} \hat{y} h \overline{y x}=\hat{x} h \bar{x}$. So, $x \leqslant{ }_{e} h$.

Let $e, j \in \operatorname{RIHom}(\alpha, \alpha)$ for some identity $\alpha$. If $e \leqslant_{e} j$, then $e=\widehat{e} j \bar{e}=j e=e j$ because the restriction idempotents commute (see Theorem 5). Therefore, $\leqslant_{e}=\leqslant_{r}=\leqslant_{l}$ on RIHom $(\alpha, \alpha)$, which we denote by $\leqslant$.

RIHom $(\alpha, \alpha)$ with $\leqslant$ forms a meet semilattice with a top element. In fact, let $e, j \in \operatorname{RIHom}(\alpha, \alpha)$. Then, 
$(e j) e=j e=e j$, by commutativity. Thus, $e j \leqslant e$. Similarly, $e j \leqslant j$. Now, let $i \leqslant e, j$. Then, $i(e j)=(i e) j=i j$ since $i \leqslant e$, and $i j=i$ since $i \leqslant j$. Thus, $i \leqslant e j$. Hence, $e \wedge j=e j$. It follows that ( $\operatorname{RIHom}(\alpha, \alpha), \leqslant)$ is a meet semilattice. The top element is $\alpha$ because if not, there is $e$ in $\operatorname{RIHom}(\alpha, \alpha)$ such that $\alpha \leqslant e$. Then, $\alpha=\alpha e$, but $\alpha e=e$ because $\alpha$ is the identity. Thus, $\alpha=e$.

The last two implications are trivial.

Proposition 6. Let $\mathscr{C}$ be a range prerestriction category. Then,

(i) $x \leqslant_{e} y \Leftrightarrow$ there exist $e_{1}, e_{2} \in R I \mathscr{C}$ such that $x=e_{1} y e_{2}$

(ii) $x \leqslant_{r} y \Leftrightarrow$ there exist $e_{1} \in R I \mathscr{C}$ such that $x=e_{1} y$

(iii) $x \leqslant_{l} y \Leftrightarrow$ there exist $e_{2} \in R I \mathscr{C}$ such that $\leqslant_{e}$

Proof. We will prove the $\leqslant_{e}$ case. Let $x=e_{1} y e_{2}$. Then, $e_{1} x=$ $x$ and $x e_{2}=x$ so that $e_{1} \hat{x}=\hat{x}$ and $\bar{x} e_{2}=\bar{x}$; hence,

$$
x=\hat{x} x \bar{x}=\hat{x} e_{1} y e_{2} \bar{x}=\hat{x} y \bar{x} .
$$

The converse is clear.

Proposition 7. Let $\mathscr{C}$ be a range prerestriction category. Then,

$$
\leqslant_{l} \circ \leqslant_{r}=\leqslant_{e}=\leqslant_{r} \circ \leqslant_{l} .
$$

Proof. Note first that $\leqslant_{r} \subseteq \leqslant_{e}$ since if $x \leqslant_{r} y$ so that $(x=\hat{x} y)$, then by $R 1$, we get $x=x \bar{x}=\hat{x} y \bar{x}$. Similarly, $\leqslant_{l} \subseteq \leqslant_{e}$. Thus, it is clear that $\leqslant_{l} \circ \leqslant_{r}$ and $\leqslant_{r} \circ \leqslant_{l}$ are contained in $\leqslant_{e}$.

Now, let $x \leqslant_{e} y$. Then, $x=\hat{x} y \bar{x}$. Put $h=\hat{x} y$. Then, by the previous proposition above, $h \leqslant_{r} y$. Furthermore, $x=h \bar{x}$ so $x \leqslant_{l} h$. Thus, we have shown $x \leqslant_{l} h \leqslant_{r} y$ giving $\leqslant_{l} \circ \leqslant_{r}=\leqslant_{e}$. The other case follows by symmetry.

The following lemma is proved in Lawson [1] for Ehresmann semigroups. We extend it to range prerestriction categories.

Lemma 4. Let $\mathscr{C}$ be a range prerestriction category. Then, $\mathscr{C}$ is a range restriction category if and only if $\leqslant_{r} \subseteq \leqslant_{l}$.

Proof. Suppose that $\mathscr{C}$ is a range restriction category. Then, $(R 4)$ is verified. If $x \leqslant_{r} y$ then $x=\widehat{x} y=\overline{\widehat{x}} y=y \overline{(\widehat{x} y)}=y \bar{x}$.

Suppose that $\leqslant_{r} \subseteq \leqslant_{l}$. Let $x$ and $y$ be two arrows. Let $h=\bar{x} y$. Then, $h \leqslant_{r} y$. Therefore, $h \leqslant_{l} y$ which gives $h=y \bar{h}=y \overline{\bar{x} y}=y \overline{x y}$.

Proposition 8. Let $\mathscr{C}$ be a range prerestriction category. Then,

(i) If $x \leqslant_{r} y$, then $x h \leqslant_{r} y h$ for all $h \in \mathscr{C}$

(ii) If $x \leqslant_{l} y$, then $h x \leqslant_{l}$ hy for all $h \in \mathscr{C}$

(iii) If $\mathscr{C}$ is a range restriction category (i.e., satisfies $R 4$ ), then $\leqslant_{e}$ is compatible with the multiplication in the sense that $x \leqslant_{e} y$, and $x^{\prime} \leqslant_{e} y^{\prime}, r\left(x^{\prime}\right)=d(x)$, and $r\left(y^{\prime}\right)=d(y)$ imply $x x^{\prime} \leqslant_{e} y y^{\prime}$
Proof. If $x \leqslant_{r} y$, then $x=\widehat{x} y$ so that $x h=\widehat{x} y h$ giving $x h \leqslant_{r} y h$. The proof of (2) is similar to (3).

By Proposition 7,

$$
\leqslant_{l} \circ \leqslant_{r}=\leqslant_{e} .
$$

By Lemma 4 , if $R 4$ holds, then $\leqslant_{r} \subseteq \leqslant_{l}$ so that

$$
\leqslant_{l} \circ \leqslant_{r} \subseteq \leqslant_{l} \circ \leqslant_{l} \subseteq \leqslant_{l},
$$

and hence, $\leqslant_{l}=\leqslant_{e}$. By part (2), we will show only that if $x \leqslant_{e} y$, then $x h \leqslant_{e} y h$ for any arrow $h$. Let $x \leqslant_{l} y$. Then, $x=y e$, where $e$ is a restriction idempotent. Thus, $x h=y e h=y h e h$. Hence, $x h \leqslant_{l} y h$.

Proposition 9. Let $\mathscr{C}$ be a range prerestriction category.

(1) If $x \leqslant r y$, then $\bar{x} \leqslant \bar{y}$ and $\hat{x} \leqslant \hat{y}$

(2) If $x \leqslant l$, then $\bar{x} \leqslant \bar{y}$ and $\hat{x} \leqslant \hat{y}$

(3) If $x \leqslant e y$, then $\bar{x} \leqslant \bar{y}$ and $\hat{x} \leqslant \hat{y}$

(4) If $x \leqslant_{r} y$ and $u \leqslant_{r} v$ and $\bar{x}=\widehat{u}$ and $\bar{y}=\widehat{v}$, then $x u \leqslant_{r} y v$

(5) If $x \leqslant_{l} y$ and $u \leqslant_{l} v$ and $\bar{x}=\widehat{u}$ and $\bar{y}=\widehat{v}$, then $x u \leqslant_{l} y v$

Proof.

(1) If $x \leqslant_{r} y$, then $x=e y$ for some restriction idempotent $e$. Thus, $\hat{x}=\hat{e y}=e \hat{y}$. Therefore, $\hat{x} \leqslant \hat{y}$. And, $\bar{x}=\overline{e y}=\overline{e y \bar{y}}=\overline{e y} \bar{y}=\overline{x y}$. Thus, $\bar{x} \leqslant \bar{y}$. The proof is similar for (2).

(3) If $x \leqslant_{e} y$, then $x=\hat{x} y \bar{x}$. Furthermore, $x=\hat{x} y \bar{x}$ implies $\bar{x}=\overline{\hat{x} y \bar{x}}=\overline{\hat{x} y} \bar{x}=\overline{\widehat{x} y \bar{y} x}=\overline{\hat{x} y} \overline{y x}=\bar{y} \overline{\hat{x} y} \bar{x}=$ $\bar{y} \bar{x} y \bar{x}=\overline{y x}$. Similarly, to this proof, we can show $\hat{x}=\hat{x} \hat{y}$. Thus, $\bar{x} \leqslant \bar{y}$ and $\hat{x} \leqslant \hat{y}$.

(4) If $x \leqslant_{r} y$ and $u \leqslant_{r} v$ and $\bar{x}=\widehat{u}$ and $\bar{y}=\widehat{v}$, then $x=\widehat{x} y$ and $u=\widehat{u} v$ so that $x u=\widehat{x} y \widehat{u} v$. Now, $\widehat{x} y=x$ so that $(\hat{x} y \bar{x}=\hat{x} y)$. However, $\bar{x}=\widehat{u}$ so that $\hat{x} y \widehat{u}=\hat{x} y$. Hence, $x u=\hat{x} y v$, giving $x u \leqslant_{r} y v$.

Proposition 10. Let $\mathscr{C}$ be a range prerestriction category. And, let e be a restriction idempotent.

(1) If $e \leqslant \hat{x}$, then there is a unique element $(e \mid x)$ such that $\widehat{(e \mid x)}=e$ and $(e \mid x) \leqslant_{r} x$

(2) If $e \leqslant \bar{x}$, then there is a unique element $(x \mid e)$ such that $\overline{(x \mid e)}=e$ and $(x \mid e) \leqslant_{l} x$

Proof. We prove (1). We will show $(e \mid x)=e x$. Note that $e x \leqslant_{r} x$ and $\hat{e x}=e \hat{x}=e$. If there exists an arrow $y$ such that $y \leqslant_{r} x$ and $\hat{y}=e$, then $y=\hat{y} x=e x$.

\section{Generalized Ehresmann Categories}

In this section, we generalize Lawson's notion of an Ehresmann category [1] and establish some helpful properties towards a Generalized Ehresmann's theorem.

First, we define a generalized Ehresmann category. 
Definition 13. Define a generalized Ehresmann category to be a category $\mathscr{C}$ equipped with two relations $\leqslant_{l}$ and $\leqslant_{r}$ on $\mathscr{C}$ satisfying the following axioms:

$\left(\mathrm{GE}_{1}\right)\left(\mathscr{C}, \leqslant_{r}\right)$ is an ordered category with corestriction. $\left(\mathrm{GE}_{2}\right)\left(\mathscr{C}, \leqslant_{l}\right)$ is an ordered category with restriction. $\left(\mathrm{GE}_{3}\right)$ Let $e$ and $j$ be in $\mathscr{C}_{0}$. Then, $e \leqslant_{r} j \Leftrightarrow e \leqslant_{l}$ jand in this case, we put $\leqslant=\leqslant_{r}=\leqslant_{l}$.

$\left(\mathrm{GE}_{4}\right)\left(\mathscr{C}_{0}, \leqslant\right)$ forms disjoint meet semilattices with a top element. Let $e$ be in $\mathscr{C}_{0}$, we denote the meet semilattice containing $e$ by $\operatorname{MSL}(e)$.

$\left(\mathrm{GE}_{5}\right) \leqslant_{r} \circ \leqslant_{l}=\leqslant_{l} \circ \leqslant_{r}$, and we define $\leqslant_{e}=\leqslant_{r} \circ \leqslant_{l}$.

$\left(\mathrm{GE}_{6}\right)$ If $x \leqslant_{r} y$, then $\operatorname{MSL}(d(x))=\operatorname{MSL}(d(y))$, and let $e$ be in $\operatorname{MSL}(d(x))=\operatorname{MSL}(d(y))$, then $(x \mid d(x) \wedge e) \leqslant_{r}$ $(y \mid d(y) \wedge e)$.

$\left(\mathrm{GE}_{7}\right)$ If $x \leqslant_{l} y$, then $\operatorname{MSL}(r(x))=\operatorname{MSL}(r(y))$, and let $e$ be in $\operatorname{MSL}(r(x))=\operatorname{MSL}(r(y))$, then $(r(x) \wedge e \mid x) \leqslant l$ $(r(y) \wedge e \mid y)$.

Proposition 11. If $\mathscr{C}$ is a range prerestriction category, then define a category $G E(\mathscr{C})$ to be

(i) Identities: all the restriction idempotents $(\alpha, e, \alpha)$ of $\mathscr{C}$, where $\alpha$ is an identity in $\mathscr{C}$.

(ii) Arrows: the arrows of $\mathscr{C}$, where $x:(\alpha, e, \alpha) \longrightarrow$ $\left(\beta, e^{\prime}, \beta\right) \Leftrightarrow e=\bar{x}$ and $e^{\prime}=\hat{x}$.

(iii) Composition denoted by $*$ : the composition $x * y$ of two elements $x$ and $y$ in $G E(\mathscr{C})$ is defined if $\hat{y}=\bar{x}$. And, when it is defined is equal to $x y$.

$\mathrm{GE}(\mathscr{C})$ with $*$ composition is a generalized Ehresmann category.

Remark 3. Observe that the morphisms of a generalized Ehresmann category are maps between idempotents. Thus, these maps satisfy the general criterion of being in the Karoubi envelope (idempotent splitting completion) of a range prerestriction category (see [11]) so that $x: e \longrightarrow e^{\prime}$ satisfies $x: e \longrightarrow e^{\prime}$ as is easily seen.

Proof. First, we have to show that $(\mathrm{GE}(\mathscr{C}))$ with $*$ is a category (i.e., $\mathrm{GE}(\mathscr{C})$ with the *-composition is a multiplicative set satisfying $C_{1}, C_{2}$, and $C_{3}$ ). We begin by showing all restriction idempotents of $\mathscr{C}$ are identities of $(\mathrm{GE}(\mathscr{C}), *)$. Let $e$ be a restriction idempotent and suppose that $e * x$ is defined for some arrow $x$. By the definition of the composition, we deduce that $\hat{x}=\bar{e}=e$. Then, $e * x=x$. Similarly, if $x * e$ is defined, then it is equal to $x$. We now check that the axioms $C_{1}, C_{2}$, and $C_{3}$ hold.

(i) Axiom $C_{1}$ holds: suppose that $x *(y * h)$ is defined. Then, $\bar{x}=\widehat{y * h}$ and $y * h$ is defined. Hence, $y * h=y h$ and $\widehat{h}=\bar{y}$. Therefore, by $R R 4$, we have $\bar{x}=\widehat{y h}=\widehat{y \hat{h}}=\widehat{y \bar{y}}=\widehat{y}$, so $x * y$ is defined. Also, $\overline{x * y}=\overline{x y}=\overline{\bar{x} y}=\overline{\hat{y} y}=\bar{y}=\widehat{h}$. Thus, $(x * y) * h$ is defined. It is clear that $(x * y) * h=x *(y * h)$. A similar argument shows that if $(x * y) * h$ exists, then $x *(y * h)$ exists and they are equal. (ii) Axiom $C_{2}$ holds: suppose that $x * y$ and $y * h$ are defined. We have that $\bar{x}=\widehat{y}$ and $\bar{y}=\widehat{h}$. Now,

$$
\widehat{y * h}=\widehat{y h}=\widehat{y \widehat{h}}=\widehat{y \bar{y}}=\widehat{y}=\bar{x} \text {. }
$$

(iii) Thus, $x *(y * h)$ is defined.

(iv)Axiom $C_{3}$ holds: for each arrow $x$, we have that $x * \bar{x}$ is defined, and we have seen that restriction idempotents are identities in $((\mathrm{GE}(\mathscr{C})), *)$. Similarly, we have $\widehat{x} * x$ is defined.

It is now clear that $((\mathrm{GE}(\mathscr{C})), *)$ is a category.

Now, $((\mathrm{GE}(\mathscr{C})), *)$ is an ordered category. From Proposition 5 , the relations $\leqslant_{r}$ and $\leqslant_{l}$ are partial orders, so $\mathrm{OC}_{1}$ is verified. And, $\mathrm{OC}_{4}$ is clear from the remark in the same Proposition. Now, by Proposition 3(1) and [12], the axiom $\mathrm{OC}_{2}$ holds and by (4) and (5) axiom $\mathrm{OC}_{3}$ holds. Proposition 4 implies the restriction and corestriction axioms. Thus, $\mathrm{GE}_{1}$ and $\mathrm{GE}_{2}$ are verified. By Proposition 5, the axiom $\mathrm{GE}_{3}$ holds. $\mathrm{GE}_{4}$ is consequence of Proposition 5. $\mathrm{GE}_{5}$ holds by Proposition 7. The axioms $\mathrm{GE}_{6}$ and $\mathrm{GE}_{7}$ holds by Proposition 8 . In fact, if $x \leqslant_{r} y$, then $\operatorname{MSL}(\bar{x})=\operatorname{MSL}(\bar{y})$, and let $e$ be in $\operatorname{MSL}(\bar{x})=\operatorname{MSL}(\bar{y})$; then, by the Proposition 8 , we have $x e \leqslant_{r} y e$; thus, $(x \mid \bar{x} \wedge e) \leqslant_{r}(y \mid \bar{y} \wedge e)$. Similarly, we can show $\mathrm{GE}_{7}$.

Thus, from a range prerestriction category, we can construct a corresponding generalized Ehresmann category. In the remainder of this section, we will show that every generalized Ehresmann category gives rise to a range prerestriction category. Let $\left(\mathscr{C}, \leqslant_{r}, \leqslant_{l}\right)$ be a fixed generalized Ehresmann category.

Lemma 5. Let $x$ and $y$ to be in $\mathscr{C}$. If $y \leqslant{ }_{e} x$, then $d(y) \leqslant d(x)$ and $r(y) \leqslant r(x)$. Furthermore, the relation $\leqslant_{e}$ is a partial order.

Proof. Let $y \leqslant_{e} x$. Then, by $\mathrm{GE}_{5}, \leqslant_{e}=\leqslant_{r} \circ \leqslant_{l}$ so that there exists an arrow $h$ such that $y \leqslant_{r} h$ and $h \leqslant_{l} x$. However, then, by $\mathrm{GE}_{1}, \mathrm{GE}_{2}$, and $\mathrm{GE}_{3}$,

$$
\begin{gathered}
d(y) \leqslant d(h), \\
r(y) \leqslant r(h), \\
d(h) \leqslant d(x), \\
(h) \leqslant r(x),
\end{gathered}
$$

so that $d(y) \leqslant d(x)$ and $r(y) \leqslant r(x)$.

Reflexivity: from $\leqslant_{r} \subseteq \leqslant_{e}$, we have $x \leqslant_{e} x$.

Antisymmetry: let $x \leqslant_{e} y$ and $y \leqslant_{e} x$ and let $h$ be an arrow such that $x \leqslant_{r} h \leqslant_{l} y$. From the first part of the lemma, we have that $d(x)=d(h)=d(y)$ and $r(x)=r(h)=r(y)$. However, $x=(r(x) \mid h)$ so that, from $r(x)=r(h)$, we obtain $x=h$. In a similar way, $h=y$.

Transitivity: let $x \leqslant_{e} y$ and $y \leqslant_{e} h$. Then, there exist arrows $u$ and $v$ such that

$$
x \leqslant_{r} u \leqslant_{l} y \leqslant{ }_{l} v \leqslant_{r} h .
$$

Thus, 


$$
x \leqslant_{r} u \leqslant{ }_{l} v \leqslant_{r} h,
$$

and there exists an arrow $u^{\prime}$ such that

$$
x \leqslant{ }_{l} u^{\prime} \leqslant_{r} v \leqslant_{r} h,
$$

but then, $x \leqslant_{l} u^{\prime} \leqslant_{r} h$ so that $x \leqslant_{e} h$.

Lemma 6. Let $x$ and $y$ be arrows in $\mathscr{C}$. Then,

(1) If $y \leqslant_{e} x$ and $d(y)=d(x)$, then $y \leqslant_{r} x$

(2) If $y \leqslant_{e} x$ and $r(y)=r(x)$, then $y \leqslant_{1} x$

(3) If $y \leqslant_{e} x$ and $d(y)=d(x)$ and $r(y)=r(x)$, then $y=x$

(4) The set MSL (e) for any identity e is an order ideal of $\left(\mathscr{C}, \leqslant_{r}\right)\left(\right.$ resp. $\left(\mathscr{C}, \leqslant_{l}\right)$ and $\left.\left(\mathscr{C}, \leqslant_{e}\right)\right)$

(5) The relation $\leqslant_{e}$ agrees with $\leqslant$ on $\mathscr{C}_{0}$

Proof.

(1) By $\left(\mathrm{GE}_{5}\right)$, there exists an arrow $h$ such that $y \leqslant_{r} h \leqslant_{l} x$; thus, by Lemma 5 ,

$$
\begin{aligned}
& d(y) \leqslant d(h) \leqslant d(x), \\
& r(y) \leqslant r(h) \leqslant r(x) .
\end{aligned}
$$

(2) $d(y)=d(h)=d(x)$. From $h \leqslant_{1} x$, we have $h=(x \mid d(h))$ by $\mathrm{GE}_{2}$; since $d(h)=d(x)$, we obtain $h=x$ giving $y \leqslant r x$.

(3) The proof of (2) is similar to (1).

(4) By (1), we have that $y \leqslant_{r} x$ so that $y=(r(y) \mid x)$ but $r(y)=r(x)$; hence, $x=y$.

(5) Let $x \leqslant_{r} e$, where $e$ is in $\mathscr{C}_{0}$. Then, $x=(r(x) \mid e)=r(x)$.

The proof of (5) is straightforward.

Lemma 7. Let $x$ be an arrow in $\mathscr{C}$. Then,

(1) If $e \leqslant d(x)$ and $j \leqslant e$, then $(x \mid j) \leqslant l(x \mid e) \leqslant l x$

(2) If $e \leqslant r(x)$ and $j \leqslant e$, then $(j \mid x) \leqslant_{r}(e \mid x) \leqslant r$

Proof. We prove (1). Note first that $j \leqslant d((x \mid e))$ so that $((x \mid e) \mid j)$ is defined and $((x \mid e) j) \leqslant(x \mid e)$. However, $((x \mid e) \mid j) \leqslant l$ and $d(((x \mid e) \mid j))=j$ so that $((x \mid e) \mid j)=$ $(x \mid j)$.

Lemma 8. Let $x$ be an arrow in $\mathscr{C}$.

(1) Let $e \in \mathscr{C}_{0}$ with $e \leqslant d(x)$. Then,

$$
(x \mid e)=\max \left\{y: y \leqslant{ }_{e} x \text { such that } d(y) \leqslant e\right\} .
$$

(2) Let $e \in \mathscr{C}_{0}$ with $e \leqslant r(x)$. Then,

$$
(e \mid x)=\max \left\{y: y \leqslant_{e} x \text { such that } r(y) \leqslant e\right\} .
$$

Proof. We will prove (1). By $\left(\mathrm{GE}_{2}\right)$, we have $(x \mid e) \leqslant l x$ and $d((x \mid e))=e$. By $\left(\mathrm{GE}_{5}\right),(x \mid e) \leqslant_{e} x$. Let $y \leqslant_{e} x$ with $d(y) \leqslant_{e} x$. By $\mathrm{GE}_{5}$, there exists an arrow $h$ such that $y \leqslant_{r} h \leqslant_{l} x$. By GE $\mathrm{G}_{6}$,

$$
(y \mid d(y) \wedge e) \leqslant_{r}(h \mid d(h) \wedge e),
$$

but, from $d(y) \leqslant e$, it follows that $d(y) \wedge e=d(y)$; thus, $y=(y \mid d(y) \wedge e)$. However, then

$$
y \leqslant_{r}(h \mid d(h) \wedge e) \leqslant_{l} h \leqslant_{l} x .
$$

From the fact that $(h \mid d(h) \wedge e) \leqslant l x$ and $(x \mid e) \leqslant l x$ and $d(h) \wedge e \leqslant e$, we have $(h \mid d(h) \wedge e) \leqslant l(x \mid e)$ by Lemma 7 . Thus, $y \leqslant_{e}(x \mid e)$ as required.

Lemma 9. Let $\mathscr{C}$ be a generalized Ehresmann category. Let $x$ and $y$ be in $\mathscr{C}$. If $y \leqslant_{e} x$, then $y=(r(y) \mid(x \mid d(y)))=$ $((r(y) \mid x) \mid d(y))$.

Proof. By Lemma 5,

$$
\begin{aligned}
& d(y) \leqslant d(x), \\
& r(y) \leqslant r(x),
\end{aligned}
$$

so that $(x \mid d(y)) \leqslant 1$. Now, $d(y) \leqslant d(x)$ so that, by Lemma 8 ,

$$
(y \mid d(x))=\max \left\{h: h \leqslant_{e} x \text { and } d(h) \leqslant d(y)\right\} .
$$

However, $y \leqslant_{e} x$ and $d(y)=d(y)$ so that $y \leqslant_{e}(y \mid d(x))$. Also, $d(y)=d(x \mid d(y))$ so that, by Lemma 6 ,

$$
y \leqslant_{r}(x \mid d(y)) \leqslant_{l} x,
$$

and hence, $y=(r(y) \mid(x \mid d(y)))$.

The proof of the other equality is similar.

Lemma 10. Let $\mathscr{C}$ be a generalized Ehresmann category. And, $x, y \in \mathscr{C}$ are two arrows.

(1) Let $d(y)=r(x)$ and let $e \leqslant d(y x)$. Then,

$$
(y x \mid e)=(y \mid r(x \mid e))(x \mid e) .
$$

(2) Let $d(y)=r(x)$ and let $e \leqslant r(y x)$. Then,

$$
(e \mid y x)=(e \mid y)(d(e \mid y) \mid x) .
$$

Proof. We will prove (1). The proof of (2) is similar. $d(y x)=$ $d(x)$ and $e \leqslant d(x)$ so that the element $(x \mid e)$ exists and $(x \mid e) \leqslant 1 x$. However, then $r((x \mid e)) \leqslant r(x)=d(y)$ so that the element $(y \mid r((x \mid e)))$ exists and $(y \mid r((x \mid e))) \leqslant l y$. Now, $(y \mid r((x \mid e)))(x \mid e)$ and $y x$ exist so that we obtain $(y \mid r((x \mid e)))(x \mid e) \leqslant_{l} y x$. However, $\quad(y x \mid e) \leqslant_{l} y x \quad$ and $d((y \mid r((x \mid e)))(x \mid e))=d((x \mid e))=e=d((y x \mid e))$ so that, by the uniqueness,

$$
(y x \mid e)=(y \mid r((x \mid e)))(x \mid e) .
$$

Proposition 12. Let $\mathscr{C}$ be generalized Ehresmann category. Define a category $R P(\mathscr{C})$ as follows: 
(i) Identities: the top elements of the meet semilattices in $\mathscr{C}$.

(ii) Arrows: if $x: d(x) \longrightarrow r(x)$ in $\mathscr{C}$, then we define an arrow in $R P(\mathscr{C})$ by $x: T_{d(x)} \longrightarrow T_{r(x)}$, where $T_{d(x)}$ and $T_{r(x)}$ are the top elements of the meet semilattices containing $d(x)$ and $r(x)$, respectively.

(iii) Composition: let $x: T_{d(x)} \longrightarrow T_{r(x)}$ assume that $y: T_{d(y)} \longrightarrow T_{r(y)}$ in $\mathscr{C}$, and $d(y)$ and $r(x)$ are in the same meet semilattice and $e=d(y) \wedge r(x)$. Therefore, $e \leqslant d(y)$ and $e \leqslant r(x)$ so that, by $G E_{1}$ and $G E_{2}$, the elements $(e \mid x)$ and $(y \mid e)$ exist. Furthermore,

$$
(y \mid e) \leqslant_{r} y,(e \mid x) \leqslant{ }_{l} x, \quad d((y \mid e))=r((e \mid x))=e .
$$

Then, the composition in $\mathscr{C}$ of $(y \mid e)(e \mid x)$ exists. We define a new composition $\otimes$ as

$$
y \otimes x=(y \mid e)(e \mid x)
$$

Then, $(R R \mathscr{C}, \otimes)$ is a range prerestriction category. To prove this, we need to show some helpful lemmas.

Lemma 11. Let $\mathscr{C}$ be a generalized Ehresmann category. And, let $x, y$ and $h$ be arrows in $\mathscr{C}$ such that $(y \otimes x) \otimes$ h exists. Then, $\quad(y \otimes x) \otimes h=(y \mid e) \otimes((e \mid y) \otimes h), \quad$ where $e=d(y) \wedge r(x)$.

Proof.

$$
\begin{aligned}
(y \otimes x) \otimes h & =((y \mid e)(e \mid x)) \otimes h=(((y \mid e)(e \mid x)) \mid i)(i \mid h), \\
& =((y \mid e) \mid r((e \mid y) \mid i))(((e \mid x) \mid i)(i \mid h)), \quad \text { where } i=d(e \mid x) \wedge r(h) \text { by the Lemma } 10 .
\end{aligned}
$$

Since $d((e \mid x)) \wedge r(h)=i$

$$
((e \mid x) \mid i)(i \mid h)=(e \mid x) \otimes h .
$$

Now, $\quad((e \mid x) \mid i) \leqslant{ }_{l}(e \mid x)$ so that $r((e \mid x) \mid i) \leqslant r((e \mid x))=e$ which means that $e \wedge r((e \mid x) \mid i)=r((e \mid x) \mid i)$. Note also that

$$
d((x \mid e)) \wedge r((e \mid x) \otimes h)=e \wedge r((e \mid x) \mid i) .
$$

This now gives

$$
(y \otimes x) \otimes h=(y \mid e) \otimes((e \mid x) \otimes h) .
$$

Remark 4. Let $e$ and $j$ be two elements of a meet semilattice of a generalized Ehresmann category. Then, if $j \leqslant e$, then there exists a unique element $(e \mid j)$ such that $(e \mid j) \leqslant e$ and $d((e \mid j))=j=d(j)$. By the uniqueness, we get that $(e \mid j)=j$. The same holds for the dual, so $(e \mid j)=j=(j \mid e)$.

Lemma 12. Let $\mathscr{C}$ be a generalized Ehresmann category. And, let $x$ be an arrow in $\mathscr{C}$.

(1) If $e \leqslant d(x)$, then $(x \mid e)=x \otimes e$

(2) If $e \leqslant r(x)$, then $(e \mid x)=e \otimes x$
Proof. We prove (1):

$$
\begin{aligned}
x \otimes e & =(x \mid d(x) \wedge e)(d(x) \wedge e \mid e) \\
& =(x \mid d(x) \wedge e)(d(x) \wedge e)(x \mid d(x) \wedge e) .
\end{aligned}
$$

However, $e \leqslant d(x)$; thus, $x \otimes e=(x \mid e)$.

Lemma 13. Let $\mathscr{C}$ be a generalized Ehresmann category. Let $x: T_{d(x)} \longrightarrow T_{r(x)}$ and $y: T_{d(y)} \longrightarrow T_{r(y)}$ be two arrows such that $y \otimes(e \otimes x)$ exists, where $e$ in $\operatorname{MSL}(r(x))=\operatorname{MSL}(d(y))$. Then, $y \otimes(e \otimes x)=(y \otimes e) \otimes x$.

Proof.

$y \otimes(e \otimes x) y \otimes((e \mid i)(i \mid x)),=y \otimes(i \mid x), \quad$ where $i=e \wedge r(x)$,

$=(y \mid j)(j \mid(i \mid x)), \quad$ where $j=d(x) \wedge i$.

However, $j \leqslant i$ so that $(j \mid(i \mid x))=(j \mid x)$; hence, $y \otimes(e \otimes x)=(x \mid j)(j \mid x)$

$$
\begin{aligned}
(y \otimes e) \otimes x & =((y \mid n)(n \mid e)) \otimes x=(y \mid n) \otimes x, \quad \text { where } n=d(y) \wedge e \\
& =((y \mid n) \mid m)(m \mid x)=(y \mid m)(m \mid x), \quad \text { where } m=n \wedge r(x), \\
(d(y) \wedge e) \wedge r(x) & =d(y) \wedge(e \wedge r(x)) .
\end{aligned}
$$

Lemma 14. Let $\mathscr{C}$ be a generalized Ehresmann category. Let $x: T_{d(x)} \longrightarrow T_{r(x)}$ and $y: T_{d(y)} \longrightarrow T_{r(y)}$ be two arrows such that $T_{r(x)}=T_{d(y)}$. Then,

(1) $r(y \otimes x)=r(y \otimes r(x))$

(2) $d(y \otimes x)=d(d(y) \otimes x)$
Proof. We will prove (1):

$r(y \otimes x)=r((y \mid e)(e \mid x))=r(y \mid e), \quad$ where $e=d(y) \wedge r(x)$,

whereas 


$$
r(y \otimes r(x))=r((y \mid e)(e \mid r(x)))=r(y \mid e) .
$$

Lemma 15. Let $\mathscr{C}$ be a generalized Ehresmann category. Let $x: T_{d(x)} \longrightarrow T_{r(x)}$ and $e$ to be in $\operatorname{MSL}(r(x))$ and $j$ in $\operatorname{MSL}(d(x))$. Then,

(1) If $u=(e \otimes x) \otimes j$, then $u \leqslant_{e} x$

(2) If $v=e \otimes(x \otimes j)$, then $v \leqslant_{e} x$

Proof. We will prove (1). Put $u^{\prime}=e \otimes x$; then, $u^{\prime}=e \otimes x=$ $((e \wedge r(x)) \mid x)$ so that $u^{\prime} \leqslant_{r} x$ by $\left(\mathrm{GE}_{1}\right)$. Also, $u=u^{\prime} \otimes j$ so that $u=\left(u^{\prime} \mid d\left(u^{\prime}\right) \wedge j\right)$ giving $u \leqslant_{l} u^{\prime}$ by $\left(\mathrm{GE}_{2}\right)$. Hence, $u \leqslant_{l} u^{\prime} \leqslant_{r} x$, giving $u \leqslant_{e} x$ by $\left(\mathrm{GE}_{5}\right)$.

Lemma 16. Let $\mathscr{C}$ be a generalized Ehresmann category. Let $x: T_{d(x)} \longrightarrow T_{r(x)}$ and $u: T_{d(u)} \longrightarrow T_{r(u)}$ be arrows. Then,

(1) If $u \leqslant_{e} x$, then $u=(r(u) \mid(x \mid d(u)))$

(2) If $u \leqslant_{e} x$, then $u=((r(u) \mid x) \mid d(u))$

Proof. We will prove (1). From $u \leqslant{ }_{e} x$, we obtain $d(u) \leqslant d(x)$ and $r(u) \leqslant r(x)$ by Lemma 5. Thus, $(x \mid d(u))$ is defined and $(x \mid d(u)) \leqslant_{l} x . \quad$ By Lemma $8, \quad u \leqslant_{e}(x \mid d(u)) \quad$ and $d(u)=d(x \mid d(u))$; thus, by Lemma $6, u \leqslant_{r}(x \mid d(u))$, where $u=(r(u) \mid(x \mid d(u)))$.

Lemma 17. Let $\mathscr{C}$ be a generalized Ehresmann category. Let $x: T_{d(x)} \longrightarrow T_{r(x)}$ and $y: T_{d(y)} \longrightarrow T_{r(y)}$ be arrows such that $T_{d(x)} \longrightarrow T_{r(x)}$ and $T_{d(y)} \longrightarrow T_{r(y)}$. Then,

(1) If $y \leqslant_{r} x$, then $y \otimes h \leqslant_{r} x \otimes h$, where $h: T_{d(h)} \longrightarrow T_{r(h)}$ and $T_{r(h)}=T_{d(x)}$

(2) If $y \leqslant_{l} x$, then $h \otimes y \leqslant l n \otimes x$, where $h: T_{d(h)} \longrightarrow T_{r(h)}$ and $T_{d(h)}=T_{r(x)}$

Proof. We will prove (1):

$$
\begin{array}{ll}
y \otimes h=(y \mid e)(e \mid h), & \text { where } e=d(y) \wedge r(h), \\
x \otimes h=(x \mid j)(j \mid h), & \text { where } j=d(x) \wedge r(h) .
\end{array}
$$

Since $y \leqslant_{r} x, \quad$ so, by $\left(\mathrm{GE}_{6}\right), \quad(y \mid d(y) \wedge r(h)) \leqslant r$ $(x \mid d(x) \wedge r(h))$ so that $(y \mid e) \leqslant_{r}(x \mid j)$. However, since $e \leqslant j$, we have $(e \mid h) \leqslant_{r}(j \mid h)$, by Lemma 7 . Now, apply $\left(\mathrm{OC}_{3}\right)$ with the order $\leqslant_{r}$ giving $(y \mid e)(e \mid h) \leqslant_{r}(x \mid j)(j \mid h)$.
Proposition 13. Let $\mathscr{C}$ be a generalized Ehresmann category. Let $x: T_{d(x)} \longrightarrow T_{r(x)}$ and let $e$ be in MSL $(r(x))$ and $j$ be in $\operatorname{MSL}(d(x))$. Then, $(e \otimes x) \otimes j=e \otimes(x \otimes j)$.

Proof. Put $u=(e \otimes x) \otimes j$. By Lemma 15 , we have $u \leqslant_{e} x$. By Lemma 16, $u=(r(u) \mid(x \mid d(u)))$. By Lemma $12, u=r(u) \otimes$ $(x \otimes d(u))$. Now,

$$
\begin{aligned}
u & =(e \otimes x) \otimes j=((e \wedge r(x)) \mid x) \otimes j \\
& =(((e \wedge r(x)) \mid x) \mid d((e \wedge r(x)) \mid x) \wedge j) \\
& \leqslant_{l}((e \wedge r(x)) \mid x)
\end{aligned}
$$

so that $r(u) \leqslant(e \wedge r(x)) \leqslant e$. Also, $d(u)=d((e \wedge r(x)) \mid x) \wedge j \leqslant j$.

And, $d(u) \leqslant j$, and we obtain $x \otimes d(u) \leqslant l$ x $\otimes j$ for

$$
\begin{aligned}
x \otimes d(u) & =(x \mid d(x) \wedge d(u)), \\
x \otimes j & =(x \mid d(x) \wedge j),
\end{aligned}
$$

and $d(u) \leqslant j$ implies $d(x) \wedge d(u) \leqslant d(x) \wedge j$ so that, by Lemma 7 ,

$$
(x \mid d(x) \wedge d(u)) \leqslant_{l}(x \mid d(x) \wedge j) .
$$

By Lemma 17 , from $(x \otimes d(u)) \leqslant_{l} x \otimes j$, we have

$$
r(u) \otimes(x \otimes d(u)) \leqslant l r(u) \otimes(x \otimes j) .
$$

However, $r(u) \leqslant e$ so that $r(u) \otimes(x \otimes j) \leqslant_{r} e \otimes(x \otimes j)$ by Lemma 7. Thus, we have shown that $(e \otimes x) \otimes j \leqslant e \otimes(x \otimes j)$.

We may similarly show by symmetry that $e \otimes(x \otimes j) \leqslant_{e}(e \otimes x) \otimes j$ and then apply the fact that $\leqslant_{e}$ is a partial order.

Lemma 18. If $e, j$ are in a meet semilattice of some generalized Ehresmann category, then $e \otimes j=e \wedge j=j \otimes e$.

Proof. $e \otimes j=(e \mid e \wedge j)(e \wedge j \mid j)=e \wedge j$

Lemma 19. Let $\mathscr{C}$ be a generalized Ehresmann category. Let $x: T_{d(x)} \longrightarrow T_{r(x)}$ and $y: T_{d(y)} \longrightarrow T_{r(y)}$ be arrows such that $T_{r(x)}=T_{d(y)}$. And, let $e$ be in MSL $(r(x))$. Then,

$$
\begin{aligned}
& \text { (1) } r((e \otimes x) \otimes y)=e \otimes r(x \otimes y) \\
& \text { (2) } d((x \otimes y) \otimes e)=d(x \otimes y) \otimes e
\end{aligned}
$$

Proof. We prove (1):

$$
\begin{aligned}
r((e \otimes x) \otimes y) & =r((e \otimes x) \otimes r(y)) \text { by Lemma } 14 \\
& =r(e \otimes(x \otimes r(y))) \text { by Proposition } 13 \\
& =r(e \otimes r(x \otimes r(y))) \text { by Lemma } 14 \\
& =r(e \otimes r(x \otimes y)) \text { by Lemma } 14 \\
& =e \otimes r(x \otimes y) \text { since } e \otimes r(x \otimes y) \text { is an identity by Lemma } 18 .
\end{aligned}
$$


Lemma 20. Let $\mathscr{C}$ be a generalized Ehresmann category. Let $x: T_{d(x)} \longrightarrow T_{r(x)}$ and $y: T_{d(y)} \longrightarrow T_{r(y)}$ be arrows such that $T_{r(x)}=T_{d(y)}$. And, let $e$ be in $M S L(r(x))$. Then, $(e \otimes x) \otimes y=e \otimes(x \otimes y)$.

Proof. Note that $e \otimes x \leqslant_{r} x$ so that, by Lemma 17,

$$
(e \otimes x) \otimes y \leqslant r x \otimes y,
$$

and hence, $(e \otimes x) \otimes y=r((e \otimes x) \otimes y) \otimes(x \otimes y)$ applying Lemma 12. By Lemma 19,

$$
r((e \otimes x) \otimes y)=e \otimes r(x \otimes y) .
$$

Thus,

$$
\begin{aligned}
(e \otimes x) \otimes y & =(e \otimes r(x \otimes y)) \otimes(x \otimes z) \\
& =e \otimes(r(x \otimes y) \otimes(x \otimes z)) \\
& =e \otimes(x \otimes y) \text { by Lemma } 13 .
\end{aligned}
$$

The proof of Proposition 12.

Proof. First, we have to show that $(R R \mathscr{C}, \otimes)$ is a category. Let us prove that top elements are identities with this new composition defined between arrows. Let $x: T_{d(x)} \longrightarrow T_{r(x)}$, so $T_{r(x)} \otimes x=\left(T_{r(x)} \mid e\right)(e \mid x)$, where $e=T_{r(x)} \wedge r(x)=r(x)$ because $\left(T_{r(x)} \wedge r(x)\right)=r(x)$ by Lemma 18. So, $T_{r(x)} \otimes x=\left(T_{r(x)} \mid e\right)(e \mid x)=r(x)(r(x) \mid x)=x$. Similarly, we can prove that $x \otimes T_{d(x)}=x$. It suffices to show that $\otimes$ is associative. Let $\mathscr{C}$ be a generalized Ehresmann category. And, let $x, y$, and $h$ be arrows in $\mathscr{C}$ such that $(y \otimes x) \otimes h$ exists. Put first $e=d(x) \wedge d(y)$ and note that

$$
\begin{aligned}
& (x \mid e)=x \otimes r(y), \\
& (e \mid y)=d(x) \otimes y,
\end{aligned}
$$

$(x \otimes y) \otimes h=(x \mid e) \otimes((e \mid y) \otimes h)$ by Lemma 11

$$
\begin{aligned}
& =(x \otimes r(y)) \otimes((d(x) \otimes y) \otimes h) \text { by the note above } \\
& =(x \otimes r(y)) \otimes(d(x) \otimes(y \otimes h)) \text { by Lemma } 20 \\
& =((x \otimes r(y)) \otimes d(x)) \otimes(y \otimes h) \text { by Lemma } 13 \\
& =(x \otimes(r(y) \otimes d(x))) \otimes(y \otimes h) \text { by Lemma } 13 \\
& =(x \otimes(d(x) \otimes r(y))) \otimes(y \otimes h) \text { by Lemma } 18 \\
& =((x \otimes d(x)) \otimes r(y)) \otimes(y \otimes h) \text { by Lemma } 13 \\
& =(x \otimes r(y)) \otimes(y \otimes h) \\
& =x \otimes(r(y) \otimes(y \otimes h)) \text { by Lemma } 13 \\
& =x \otimes((r(y) \otimes y) \otimes h) \text { by Lemma } 20 \\
& =x \otimes(y \otimes h) .
\end{aligned}
$$

Now, for every arrow $x$ in $\mathscr{C}$, we will write $\bar{x}=d(x)$ and $\widehat{x}=r(x)$. We have to prove the eight axioms for range prerestriction category:

(R1) $x \otimes \bar{x}=x$ for each map $x$

$(R 2) \bar{x} \otimes \bar{y}=\bar{y} \otimes \bar{x}$ for all maps $x, y$ with $\bar{x}$ and $\bar{y}$ are in the same meet semilattice
(R3) $\overline{y \otimes \bar{x}}=\bar{y} \otimes \bar{x}$ for all maps $x, y$ with $\bar{x}$ and $\bar{y}$ are in the same meet semilattice

$(p R 4) \overline{y \otimes x}=\bar{y} \otimes x$ for all maps $x, y$ with $\hat{x}$ and $\bar{y}$ are in the same meet semilattice

$(R R 1) \overline{\hat{x}}=\hat{x}$ for each map $x$

$(R R 2) \hat{x} \otimes x=x$ for each map $x$

$(R R 3) \widehat{y \otimes x}=\bar{y} \otimes \hat{x}$ for all maps $x, y$ with $\hat{x}$ and $\bar{y}$ are in the same meet semilattice

$(R R 4) \widehat{y \otimes \hat{x}}=\widehat{y \otimes x}$ for all maps $x, y$ with $\widehat{x}$ and $\bar{y}$ are in the same meet semilattice

$R 1$ and $R R 2$ are immediate. To prove $R 2$, we use Lemma 18. We can prove $p R 4$ and $R R 4$ using Lemma 14 . $R R 1$ comes from the definition of restriction idempotent. To prove $R 3$, we have to use a particular case of $p R 4 \overline{x \otimes y}=\overline{\bar{x} \otimes y}$ (which is already proved) when $y=\bar{y}$; thus, $\overline{x \otimes \bar{y}}=\overline{\bar{x} \otimes \bar{y}}$. Then, $\overline{\bar{x} \otimes \bar{y}}=\bar{x} \otimes \bar{y}$ because $\bar{x} \otimes \bar{y}=\bar{x} \wedge \bar{y}$ by Lemma 18 . However, $\bar{x} \wedge \bar{y}$ is a restriction idempotent; thus, $\bar{x} \wedge \bar{y}=\overline{\bar{x}} \wedge \bar{y}$. Therefore, $R 3$ holds. It remains to show $R R 3 \widehat{\bar{y} \otimes x}=\bar{y} \otimes \widehat{x}$, for which we are going to use $R R 4$. By $R R 4$, we have $\widehat{y \otimes x}=\widehat{y \otimes \hat{x}}$ which is equal to $\bar{y} \otimes \widehat{x}$ for the same reason as above.

\section{A Generalization of Ehresmann's theorem}

Now, we will define a functor between range prerestriction categories.

Definition 14. Let $\mathcal{S}$ and $\mathscr{T}$ be two range prerestriction categories. Let $\theta$ be functor from $\delta$ to $\mathscr{T}$. Then, $\theta$ is called a functor of range prerestriction categories if $\theta(\bar{x})=\overline{\theta(x)}$ and $\theta(\widehat{x})=\widehat{\theta(x)}$.

Remark 5. If $\mathscr{C}$ is a generalized Ehresmann category, then $R P(\mathscr{C})$ is a range prerestriction category. In addition, the orders $\leqslant_{l}$ and $\leqslant_{r}$ in $R P(\mathscr{C})$ coincide with the generalized Ehresmann category orders.

Proof. Suppose that $x \leqslant_{l} y$ in $R P(\mathscr{C})$. Then, for some $e$ in $\mathscr{C}_{0}$, we have $x=y \otimes e$ which is equals $(y \mid d(y) \wedge e)$. We have shown therefore that $x \leqslant_{1} y$ in $\mathscr{C}$.

Conversely, let $x \leqslant_{l} y$ in $\mathscr{C}$. Then, $x=(y \mid d(x))$ which equals $y \otimes d(x)$. Thus, we have shown that $x \leqslant_{l} y$ in $R P(\mathscr{C})$.

Now, we define a functor between generalized Ehresmann categories.

Definition 15. Let $\mathscr{C}$ and $\mathscr{D}$ be two generalized Ehresmann categories. Let $F$ be a functor between $\mathscr{C}$ to $\mathscr{D}$. Then, $F$ is called a strongly ordered functor if

(1) If $x \leqslant_{l} y$ (resp. $x \leqslant_{r} y$ ), then $F(x) \leqslant_{l} F(y)$ (resp. $\left.F(x) \leqslant{ }_{r} F(y)\right)$

(2) If $e, j \in \mathscr{C}_{0}$ and $e$ and $j$ are in the same meet semilattice, then $F(e \wedge j)=F(e) \wedge F(j)$

Lemma 21. Let $\theta: \mathcal{S} \longrightarrow \mathscr{T}$ be a functor of range prerestriction categories. Define $G E(\theta): G E(\mathcal{S}) \longrightarrow G E(\mathscr{T})$ by 
$G E(\theta)(x)=\theta(x)$, where $x$ is an arrow in $G E(\mathcal{S})$. Then, $G E(\theta)$ is a strongly ordered functor.

Proof. The proof is straightforward.

Lemma 22. Let $F: \mathscr{C} \longrightarrow \mathscr{D}$ be a strongly ordered functor. Define $R P(F): R P(\mathscr{C}) \longrightarrow R P(\mathscr{D})$ by $R P(F)(x)=F(x)$, where $x$ is an arrow in $R P(\mathscr{C})$. Then, $R P(F)$ is a functor of range prerestriction categories.

Proof. Let $x, y \in \mathrm{RP}(\mathscr{C})$ such that $T_{r(y)}=T_{d(x)}$; then, by definition

$$
\mathrm{RP}(F)(x \otimes y)=F(x \otimes y)=F((x \mid e)(e \mid y))=F(x \mid e) F(e \mid y),
$$$$
\text { where } e=d(x) \wedge r(y) \text { because } F \text { is a functor. }
$$

Now, $(x \mid e) \leqslant l$, since $F$ is strongly ordered, and we have $F(x \mid e) \leqslant{ }_{l} F(x)$. Moreover, $d(x \mid e)=e$; therefore, $d(F(x \mid e))=$ $F(d(x \mid e))=F(e)$. Now, from the fact that $F(x \mid e) \leqslant{ }_{l} F(x)$ and $d(F(x \mid e))=F(e)$, we have $F(e) \leqslant d(F(x))$; thus, the element $(F(x) \mid F(e))$ exists. By the uniqueness, we get that $F(x \mid e)=(F(x) \mid F(e))$. Similarly, $\quad F(e \mid y)=(F(e) \mid F(y))$. Thus,

$$
\mathrm{RP}(F)(x \otimes y)=(F(x) \mid F(e))(F(e) \mid F(y)) .
$$
so

However, $\quad F(e)=F(d(x) \wedge r(y))=d(F(x)) \wedge r(F(y))$,

$$
\mathrm{RP}(F)(x \otimes y)=F(x) \otimes F(y)=\mathrm{RP}(F)(x) \otimes \mathrm{RP}(F)(y) .
$$

Finally, $\quad \operatorname{RP}(F)(\bar{x})=F(\bar{x})=F(d(x))=d(F(x))=$ $\overline{\mathrm{RP}(F)(x)}$. Similarly, we can prove $\operatorname{RP}(F)(\widehat{x})=\widehat{\mathrm{RP}(F)}$ $(x)$.

Theorem 6. The category of range prerestriction categories and functors of range prerestriction categories is isomorphic to the category of generalized Ehresmann categories and strongly ordered functors.

Proof. We already proved that there is a functor from any range prerestriction category to generalized Ehresmann category and there is a functor opposite to the direction. To prove the isomorphism, we need to show that $\mathrm{RP} \circ \mathrm{GE}$ and $\mathrm{GE} \circ \mathrm{RP}$ are identity functors in each case. This now is straightforward.

\section{Range Restriction Categories}

In this section, we will present a special case of what we proved in Theorem 1. We will start our section by an equivalent definition of range restriction category.

Definition 16. A range prerestriction category with $R 4$ $(\bar{y} x=x \overline{y x}$, where $r(x)=d(y))$ is called a range restriction category.

It is easy to see that this definition is equivalent to Guo's definition [4]. Therefore, the order $\leqslant_{r}$ is included in the order $\leqslant_{l}$ as proved in Lemma 4 . From this remark, we will give another definition for generalized Ehresmann category called generalized $R_{4}$-Ehresmann category which is defined as follows.

Definition 17. Define a generalized $R_{4}$-Ehresmann category to be an Ehresmann category with additional condition $\mathrm{GE}_{5}^{\prime}$ $\leqslant_{l}=\leqslant_{r} \circ \leqslant_{l}=\leqslant_{l} \circ \leqslant_{r}$

We can deduce the proposition below which is another version of Ehresmann's theorem but now for a range restriction category.

Proposition 14. The category of range restriction categories and functors of range restriction categories is isomorphic to the category of generalized $R_{4}$-Ehresmann categories and strongly ordered functors.

Proof. The proof is straightforward.

\section{Inverse Categories}

Inverse categories are defined in [9], see also [13]. In this section, we will give a proposition which is again a special case of Theorem 1, and a categorical version of the standard Ehresmann's theorem for inverse semigroups. This result was first proved by Lawson in [14].

Proposition 15. Let $\mathscr{C}$ be a category. Then, the following are equivalent:

(1) $\mathscr{C}$ is an inverse category (i.e., every arrow $x: \alpha \longrightarrow \beta$ has a unique $y: \beta \longrightarrow \alpha$ with $x y x=x$ and $y x y=y$ ).

(2) $\mathscr{C}$ is a range prerestriction category in which each map is a partial isomorphism (i.e., there exist $x^{-1}$ such that $x^{-1} x=\bar{x}$ and $\left.x x^{-1}=\overline{x^{-1}}\right)$. Moreover, $\mathscr{C}$ is range restriction.

Proof. (1) implies (2) is immediate.

(2) implies (1). First, we are going to show that if a range prerestriction category has a partial isomorphism, then $\mathscr{C}$ is range restriction category. It suffices to prove, in fact, $\bar{y} x=$ $y^{-1} y x=y^{-1} y x f^{-1} x=f f^{-1} y^{-1} y x=x(y x)^{-1} y x=x \overline{y x}$.

It remains to that $x^{-} 1$ is unique to prove the equivalence. Suppose that $x$ has two inverses $g$ and $h$; thus, $h f=\bar{x}, f h=\bar{h}, g x=\bar{x}$, and $x g=\bar{g}$. Therefore, $g=g \bar{g}=$ $g \times g=h x g=h x g \times g=g \times h \times g=g x g \times h=\bar{x} h=h f h=h$.

Now, if generalized $R_{4}$-Ehresmann category satisfies that every arrow has an inverse, then we shall call it a general ordered groupoid.

Definition 18. Define general ordered groupoid to be a category $\mathscr{C}$ equipped with a relation $\leqslant$ satisfying the following axioms:

$\mathrm{GOG}_{1} \mathscr{C}$ is an ordered category with corestriction. $\mathrm{GOG}_{2} x \leqslant y \Rightarrow x^{-1} \leqslant y^{-1}$

$\mathrm{GOG}_{3} \forall x, y, u$ and $v \in \mathscr{C}$ such that $x \leqslant y$ and $u \leqslant v$ and also the products $x u$ and $y v$ are defined; then, $x u \leqslant y v$. 
$\mathrm{GOG}_{4}\left(\mathscr{C}_{0}, \leqslant\right)$ forms disjoint meet semilattices with a top element. Let $e$ be in $\mathscr{C}_{0}$, we denote the meet semilattice containing $e$ by $\operatorname{MSL}(e)$.

Proposition 16. The category of the inverse categories and functors of range prerestriction categories is isomorphic to the category of general ordered groupoids and strongly ordered functors.

Proof. The proof is straightforward.

\section{Data Availability}

No data were used to support the findings of the study.

\section{Disclosure}

This paper is a part of the author's master thesis.

\section{Conflicts of Interest}

The author declares that there are no conflicts of interest.

\section{Acknowledgments}

The author would like to thank his supervisor, Philip J. Scott, for all his support and the freedom when doing the research. The author would like to thank his ex-Ph.D supervisor, Benjamin Steinberg, for his guidance while writing this research article.

\section{References}

[1] M. V. Lawson, "Semigroups and ordered categories. I. The reduced case," Journal of Algebra, vol. 141, no. 2, pp. 422-462, 1991.

[2] V. Gould, "Restriction and ehresmann semigroups," 2010.

[3] V. Gould, "Notes on restriction semigroups and related structures; formerly (weakly) left e-ample semigroups," 2010.

[4] X. Guo, "Restrictions, ranges, partial map categories, and fibrations," 2004.

[5] B. Schweizer and A. Sklar, "Function systems," Mathematische Annalen, vol. 172, no. 1, pp. 1-16, 1967.

[6] W. Hajji, "Generalization of ehresmann's theorem," 2005.

[7] G. M. S. Gomes and V. Gould, "Fundamental Ehresmann semigroups," Semigroup Forum, vol. 63, no. 1, pp. 11-33, 2001.

[8] E. Manes, Cockett-Lack Restriction Categories, Semigroups and Topologies, FMCS, Ottawa, UK, 2003.

[9] J. R. B. Cockett and S. Lack, "Restriction categories I: categories of partial maps," Theoretical Computer Science, vol. 270, no. 1-2, pp. 223-259, 2002.

[10] E. Manes and J. R. B. Cockett, Classical Restriction Categories, Preprint, London, UK, 2005.

[11] J. Lambek and P. Scott, Introduction to Higher-Order Categorical Logic, Cambridge University Press, London, UK, 1988.

[12] C. Ehresmann, Catégories et structures, Dunod, Paris, UK, 1965.

[13] J. Kastl, "Inverse categories, algebraische modelle, kategirien, und gruppoide (=stud.algebra anwendungen 7)," 1979.

[14] M. V. Lawson, "Inverse categories and ordered groupoids," UCNW Maths Preprints, vol. 91, p. 26, 1998. 